\title{
Detection of Carbapenemase Production by Rapidec Carba NP Test among Gram Negative Isolates in a Tertiary Care Hospital
}

\author{
Sabina Ansari ${ }^{1 *}$, Maimoona Mustafa ${ }^{2}$, Md Khaleel $^{3}$
}

\author{
${ }^{1}$ Post graduate, Department of Microbiology, Deccan College of Medical Sciences, Hyderabad, Telangana, India \\ ${ }^{2}$ Professor, HOD, Department of Microbiology, Deccan College of Medical Sciences, Hyderabad, Telangana, India \\ ${ }^{3}$ Professor, Department of Microbiology, Deccan College of Medical Sciences, Hyderabad, Telangana, India
}

DOI: $10.36347 /$ sjams.2020.v08i12.025

| Received: 14.12.2020 | Accepted: 22.12.2020 | Published: 24.12.2020

*Corresponding author: Sabina Ansari

\section{Abstract}

Original Research Article

Introduction: The spread of carbapenemase producers by Gram negative bacteria has been a cumbersome issue. Several phenotypic and genotypic tests are present for detection of carbapenemases but are time consuming, require expertise and well established laboratory. This study aims at detection of carbapenemase production by using Rapid turnaround time test Rapidec Carba NP. This test is based on the detection of carbapenem hydrolysis by carbapenemase producing bacteria. Hydrolysis acidifies the medium which results in color change of the $\mathrm{pH}$ indicator. Materials and Methods: A prospective study of six months duration was done among 150 gram negative bacterial isolates obtained from various clinical samples in a tertiary care hospital. Resistance to carbapenem was detected using Meropenem disk $(10 \mu \mathrm{g})$ by kirby bauer disc diffusion method. Rapidec Carba NP test were carried out on all these carbapenem-resistant GNB isolates. Results: Among 150 Gram negative isolates 29(19\%) showed resistance to Meropenem were subjected to Rapidec carba np. 93\% (27/29) showed the presence of carbapenemase by Rapidec Carba NP test. Among the carbapenem resistant GNB isolates, 48.27\% (14/29) were Klebsiella pneumoniae, $24.13 \%$ (7/29) were Escherichia coli, 20.68\% (6/29) were Pseudomonas aeruginosa and 6.89\% (2/29) were Acinetobacter baumanni. Conclusion: Rapidec Carba NP was sensitive enough to confirm carbapenemase producing isolates within a short time of less than $<2 \mathrm{~h}$ accurately.

Keywords: Carbapenemases, Gram negative bacteria, Rapidec carba np.

Copyright $(\mathcal{C}) 2020$ The Author(s): This is an open-access article distributed under the terms of the Creative Commons Attribution 4.0 International License (CC BY-NC 4.0) which permits unrestricted use, distribution, and reproduction in any medium for non-commercial use provided the original author and source are credited.

\section{INTRODUCTION}

Carbapenemases are $\beta$-lactamases that hydrolyze penicillins, in most cases cephalosporins, to varying degrees carbapenems and monobactams [1]. Carbapenemase production in gram negative bacilli results in resistance to carbapenems and which poses a major challenge to the health-care system, by limiting the options of antibiotics available to treat these infections [2]. A variety of carbapenemases has been reported in Gram negative bacteria such as KPC (Ambler Class A), metallo- $\beta$-lactamases (MBL) of VIM-, IMP- and NDM (New Delhi MBL)-type (Ambler Class B), and OXA-48-types (Ambler Class D).Detection of Gram negative bacteria (GNB) that produce carbepenemase has epidemiological significance in controlling further transmission. Laboratory strategies for carbapenemase detection consist of routine AST and a confirmatory test.

Molecular detection of carbapenemase genes are time consuming and [3-6] expensive require expertise and well established laboratory.
Clinical and Laboratory Standards Institute (CLSI) recommended two phenotypic methods Modified Hodge test (MHT) and Carba NP test as the confirmatory test [7]. As Modified Hodge test lacks specificity and there is delay in obtaining the results (24-48 h) after isolation of a bacterial colony [8].

The present study was undertaken to evaluate turnaround time test Rapidec Carba NP for detection of carbapenemase producers. The test is based on hydrolysis of the $\beta$-lactam ring of carbapenem, was described by Patrice Nordmann, Laurent Poirel, and Laurent Dortet [9]. The principle of the test was that in vitro hydrolysis of a carbapenem leads to change of $\mathrm{pH}$ (decreased $\mathrm{pH}$ ) that brings about visible color change of the medium from red to yellow/light orange with phenol red indicator.

\section{Material and Methods}

A prospective study was conducted for a period of six months at Department of Microbiology of a tertiary care teaching hospital from June to December 
2019 after obtaining Ethical clearance from Institutional Ethics Committee.

Clinical samples such as urine, pus, blood, sputum, and tracheal aspirates submitted to Microbiology laboratory for routine diagnostic workup were processed and AST was performed by standard Microbiological techniques [10].

GNB isolates which showed inhibition zone diameter of $\leq 19 \mathrm{~mm}$ to Meropenem (10mcg) were included for the study. Among a total of $150 \mathrm{GNB}$ isolates, 29 which showed resistance to Meropenem were subjected to Rapidec carba np test.

\section{Rapidec carba np test}

\section{Preparation of the strip}

Remove the strip from its packaging.

Write the specimen reference numbers on the strip.

\section{Preparation of the test}

Open an ampule of API suspension Medium (2ml) as per manufactures.

Dispense $100 \mu \mathrm{L}$ into each of the wells a, b and c

Place a lid on the strip

Leave for 4-5 minutes at room temperature (15-25c) and then

Gently mix the contents of well $\mathrm{b}$ using a stick provided in the kit.

\section{Preparation of the inoculum and bacterial lysis}

Place the strip on the two colored (black and white) support. Position wells band $\mathrm{c}$ on the black background to facilitate comparison of turbidities.

With the end of a new stick, pick up several colonies of the same morphology taking care not to pick up any fragments of agar.
Deposit the contents of the stick in well $\mathrm{c}$ and mix. Repeat this step several until a turbidity equivalent to that of well $b$ is obtained.

The suspension in well c must be perfectly homogenous, with no aggregates and the bottom of the tube must not be visible.

Leave for 30 minutes at room temperature

\section{Procedure}

Transfer $25 \mu \mathrm{l}$ from well c to wells $\mathrm{d}$ and e then,

Transfer $25 \mu \mathrm{l}$ from well a to wells $\mathrm{d}$ and e

Incubate the strip for 30-40 minutes at 33-38c

Place the strip on the black and white support. Position wells $\mathrm{d}$ and $\mathrm{e}$ on the white background to facilitate reading. Perform the initial reading.

In case of a negative or doubtful reaction, reincubate the strip and perform a second reading 11/2 hours later. The total test incubation time must not exceed 2 hrs.

\section{Reading the strip}

Place the strip on the two colored (black and white) support. Position wells $\mathrm{d}$ and e on the white background to facilitate reading.

Reading is performed by comparing the colors in wells $\mathrm{d}$ and e, ensuring that the strip is firmly pressed against the support.

A test is positive when a significant variation in color is observed between the two wells.

Table-1: strip reading of Rapidec carba np

\begin{tabular}{|l|l|l|}
\hline Control well (d) & Test well (e) & Interpretation \\
\hline Red & Red & Negative \\
\hline Orange & Orange & \\
\hline Red & Yellow, light orange, orange, dark orange & \multirow{2}{*}{ Positive } \\
\cline { 1 - 2 } Orange & Yellow & \multirow{2}{*}{ Un interpretable } \\
\hline Any color other than red or orange & Not applicable & \\
\hline Orange & Red & \\
\cline { 1 - 2 }
\end{tabular}

\section{Quality control}

The media, strips and reagent are systematically quality controlled at various stages of their manufacturer.
Klebsiella pneumoniae ATCC 700603 is used as negative control. 


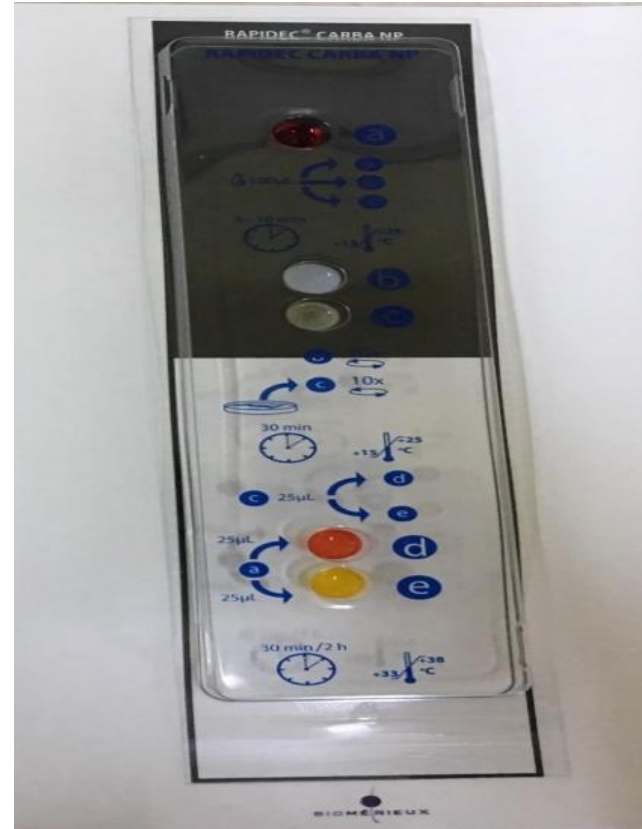

Fig-1: Positive Rapidec Carba NP test showing colour changed from Red in Control well $d$ to Yellow in Reaction well e

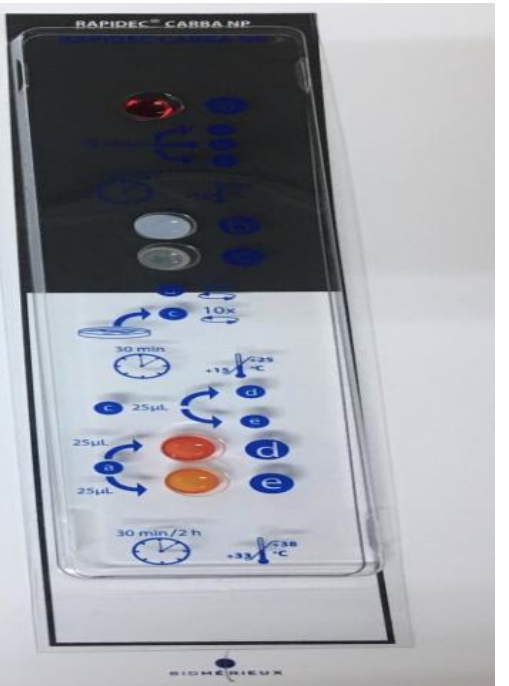

Fig-2: Positive Rapidec Carba NP test showing colour changed from Red in control well d to orange in Reaction well e

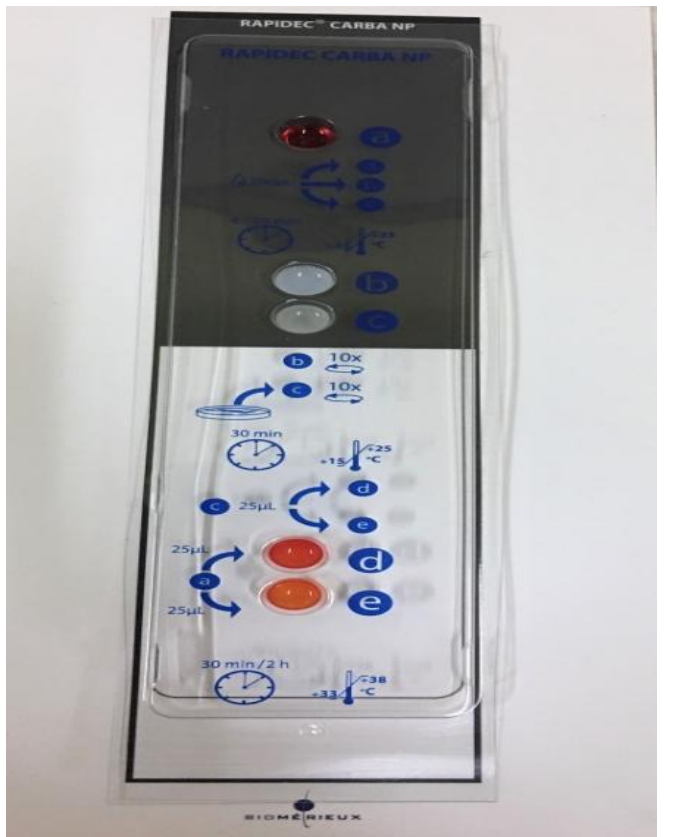

Fig-3: Negative Rapidec Carba NP test with no colour change From Red in control well d to Red in reaction well $\mathrm{e}$

\section{Results}

A total of 150 well-characterized GNB were isolated during the study period, out of which $19 \%$ (29/150) isolates showed resistance to Meropenem on routine AST. Among the carbapenem resistant GNB isolates, 48.27\% (14/29) were Klebsiella pneumoniae, $24.13 \%$ (7/29) were Escherichia coli, $20.68 \%$ (6/29) were Pseudomonas aeruginosa and $6.89 \% \quad(2 / 29)$ were Acinetobacter baumanni. Maximum number of sample was urine $12(41.3 \%)$ followed by pus 7 (24.14\%), Sputum 6 (20.68\%) and blood 4 (13.8\%). Out of the $29 \mathrm{GNB}$ isolates found to be resistant, $93 \%$ (27/29) showed the presence of carbapenemase activity by Rapidec Carba Np test.

Table-2: Gram-negative bacilli isolate showing results for Rapidec Carba np test

\begin{tabular}{|l|l|}
\hline \multicolumn{1}{|c|}{ GNB strains } & Rapidec carba np test Positive \\
\hline Klebsiella pneumoniae & $13 / 14(92.8 \%)$ \\
\hline Escherichia coli & $7 / 7(100 \%)$ \\
\hline Pseudomonas aeruginosa & $5 / 6(83.33 \%)$ \\
\hline Acinetobacter baumanni & $2 / 2(100 \%)$ \\
\hline Total & $27 / 29(93 \%)$ \\
\hline
\end{tabular}

\section{DISCUSSION}

Resistance to different carbapenems is increasing globally at a high speed, leading us to a situation when virtually no antibiotic will be effective for infections caused by carbapenemase producing bacteria. In this study, the prevalence of carbapenem resistant bacteria was $19.33 \%$. High prevalence of resistance to carbapenems has been reported from different parts of India [11, 12]. High prevalence of 
carbapenem resistance was reported in Klebsiella spp. (31\%-51\%), Pseudomonas species (39\%-59\%), and E. coli $(2 \%-13 \%)$, isolated from wards and ICU of a tertiary hospital in Delhi ranging from $17 \%$ to $22 \%$ [13], between $13 \%$ and 59\%[12]. Another study by Gupta et al. [13] showed the prevalence of carbapenem resistance in different GNB ranging from $17 \%$ to $22 \%$. Most of the resistance to carbapenems in our institute were observed in K. pneumoniae (48.27\%), followed by E. coli $(24.13 \%)$ and P. aeruginosa $(20.68 \%)$.

Maximum number of sample was urine 21 (41\%) followed by pus $7(24.54 \%)$, sputum $6(20 \%)$ and blood $(10.3 \%)$ obtained in this study. A comparable study in north India [14], most of the carbapenem resistant organism was isolated from urine $47.1 \%$ $(n=20)$ followed by pus $27.1 \%(n=13)$. Nagaraj $S$ et al. [15], had comparable findings where they observed that the carbapenem-resistant organisms were isolated mainly from urine samples up to $42 \%(\mathrm{n}=21)$, followed by wound discharge $18 \%$ and respiratory secretions $16 \%$.

In this study, Rapidec Carba np test were performed individually without knowing the genotype of the strains. Among the isolates which showed resistance to meropenem, 93\% (27/29) were positive for Rapidec Carba NP test. The limitation in the study is that the enzymes for resistance to carbapenems could not be identified and differentiated.

\section{CONCLUSION}

Detection of carbapenemase-producing bacteria has great impact on hospital infection control and for epidemiological purpose for preventing further transmission of resistance. In this study, it was shown that the Rapidec Carba NP is a rapid test where the results can be read in short time. This test shows visible color change leading to simple interpretation. It is easy to perform in clinical laboratory, no costly reagent or special instrument required, and no special skill or training required.

\section{REFERENCES}

1. Giske CG, Martinez-Martinez L, Cantón R, Stefani S, Skov R, Glupczynski Y. EUCAST Guidelines for Detection of Resistance Mechanisms and Specific Resistances of Clinical and/or Epidemiological Importance 2013. EUCAST; 2013.

2. Nordmann P, Dortet L, Poirel L. Carbapenem resistance in Enterobacteriaceae: Here is the storm! Trends Mol Med 2012; 18:263-72.
3. Nordmann P, Naas T, Poirel L. Global spread of carbapenemase producing Enterobacteriaceae. Emerg Infect Dis. 2011; 17:1791-9.

4. Nordmann P, Naas T, Poirel L. Global spread of carbapenemase producing Enterobacteriaceae. Emerg Infect Dis. 2011; 17:1791-9.

5. Nordmann P, Gniadkowski M, Giske CG, Poirel L, Woodford N, Miriagou V. European Network on Carbapenemases. Indentification and screening of carbapenemase producing Enterobacteriaceae. Clin Microbiol Infect. 2012; 18:432-440.

6. Centers of Disease Control and Prevention. Detection of Enterobacteriaceae isolates carrying metallo-lactamases - United States. MMWR Morb Mortal Wkly Rep. 2010; 59:750-750.

7. Nordman P, Poirel L, Carrier A, Ma T, Walsh TR. how to detect NDM- producers? J Clin Microbiol. 2011; 49:718-39.

8. Clinical and Laboratory Standards Institute. Performance Standards for Antimicrobial Susceptibility Testing; $25^{\text {th }}$ Informational Supplement. CLSI Document M100-S25. Wayne PA: Clinical and Laboratory Standards Institute; 2015.

9. Girlich D, Poirel L, Nordmann P. Value of the modified Hodge test for detection of emerging carbapenemases in Enterobacteriaceae. J Clinical Microbiology. 2012; 50:477-9.

10. Nordmann P, Poirel L, Dortet L. Rapid detection of carbapenemase producing Enterobacteriaceae. Emerg Infect Dis. 2012; 18:1503-1510.

11. Collee JG, Digcid JP, Fraser AG, Mackie and MacCartney. Practical medical Microbiology $14^{\text {th }}$ edition (Churchill living stone Edinburgh). 1996:413-418.

12. Taneja N, Maharwal S, Sharma M. Imipenem resistance in nonfermenters causing nosocomial urinary tract infections. Indian $\mathrm{J}$ Med Science. 2003; 57:294-9.

13. Wattal C, Goel N, Oberoi JK, Raveendran R, Datta S, Prasad KJ. Surveillance of multidrug resistant organisms in tertiary care hospital in Delhi, India. J Associate Physicians India. 2010; 58:32-6.

14. Gupta E, Mohanty S, Sood S, Dhawan B, Das BK, Kapil A. Emerging resistance to carbapenems in a tertiary care hospital in North India. Indian J Med Res. 2006; 124:95-8.

15. Mate H, Sulochana D, Mamta D. Prevalence of carbapenem resistance among gram-negative bacteria in a tertiary care hospital in North-East India. J Dent Med Sci. 2014; 13(12):56-60.

16. Nagaraj S, Chandran SP, Shamanna P, Macaden R. Carbapenem resistance among Escherichia coli and Klebsiella pneumoniae in a tertiary care hospital in South India. Indian J Med Microbiol. 2012; 30(1):93-5. 